\title{
Modeling computerized physician order entry with decision support system for diabetic patients
}

\author{
Leila Shahmoradi1(i), Marjan Ghazi Saeedi1(i), Safieh Ilati Khangholi ${ }^{1}$, Arezoo Dehghani \\ Mahmodabadi ${ }^{*}$ (D)
}

1Department of Health Information Management, Faculty of Allied Medical Sciences, Tehran University of Medical Sciences, Tehran, Iran ${ }^{2}$ Department of Health Information Technology, School of Public Health, Shahid Sadoughi University of Medical Sciences, Yazd, Iran

\begin{tabular}{ll}
\hline Article Info & A B S T R A C T \\
\hline $\begin{array}{l}\text { Article type: } \\
\text { Research }\end{array}$ & Introduction: Providing care for patients and preventing complications is \\
& one of the major subjects in medical sciences. Computerized Physician \\
& Order Entry (CPOE) with a decision support system is expected to deliver \\
Article History: & many benefits. A system with decision support system may help clinicians, \\
Received: $2019-10-07$ & patients, and others to suggest patient-appropriate evidence-based \\
Accepted: $2019-12-01$ & treatment options. The present study was conducted to prepare a \\
Published: $2020-03-04$ & conceptual model for a CPOE system of diabetic patients (Type 2) using \\
& Unified Modeling Language (UML). Then, a software program was designed \\
* Corresponding author: & accordingly. \\
Arezoo Dehghani Mahmodabadi &
\end{tabular}

Arezoo Dehghani Mahmodabadi

Department of Health Information Technology, School of Public Health, Shahid Sadoughi University of Medical Sciences, Yazd, Iran

Email:

dehghani.arezoo123@gmail.com

\author{
Keywords: \\ Computerized Physician Order Entry \\ System \\ Type 2 Diabetes Mellitus \\ $U M L$ \\ Minimum Data Set \\ Computer Systems Development
}

Material and Methods: This cross-sectional study was conducted in 2017. A minimum data set of patient records was used as the patient profile in the system, and a list of drugs and functional requirements of the CPOE system for diabetic patients was provided. Following the confirmation of the minimum data set by diabetes specialists, UML figures were drawn and the software was designed.

Results: The minimum data set of patient records included demographic and clinical information as well as laboratory tests. Functional requirements of the $\mathrm{CPOE}$ system for type 2 diabetic patients consisted of the possibility of recording simple and complicated orders, connecting the system to the pharmacy or other auxiliary information systems, controlling drug side effects, etc.

Conclusion: A CPOE system should have minimum errors in documentations and provide information on allergies, drug interactions, and side effects in a timely manner to reduce medical errors, especially drug errors, increase physician efficiency and patient satisfaction, and finally promote the quality of healthcare services.

Cite this paper as:

Shahmoradi L, Ghazi Saeedi M, Ilati Khangholi S, Dehghani Mahmodabadi A. Modeling Computerized Physician Order Entry with Decision Support System for Diabetic Patients. Front Health Inform. 2020; 9: 26. DOI: 10.30699/fhi.v9i1.206

\section{INTRODUCTION}

Providing care for patients and preventing complications is one of the major subjects in medical sciences. Most medical errors are associated with drug errors, which is the most common threat to patient safety [1]. On the other hand, it includes all phases of ordering, prescribing, drug distributing, and managing drug consumption. Improvement of the medical and clinical order process using computerized physician order entry (CPOE) systems is one of the applications of information technology that leads to reduced medical errors and drug side effect costs and improves drug consumption and management [2-ㅍ]. Current studies show different percent of medication error in any phase of medication process []․ CPOE is an electronic process allowing users to enter orders electronically for easy management of the results of their orders $[\underline{6}, \underline{7}]$.

Improvement of the drug ordering process with CPOE systems is an example of information technology. These systems reduce serious medical errors and costs of drug side effects, and improve drug management through standardizing the processes, instructing the physicians on ordering time, suggesting appropriate dosages, and check for allergies and drug-drug interactions $[\underline{5}, \underline{6}, \underline{8}-\underline{11}]$.

A CDSS is a computerized system that assists clinicians 
in assessing disease status, in making a diagnosis, in selecting appropriate therapy or in making other clinical decisions. Moreover, CPOE systems and clinical decision support systems (CDSSs) may reduce medication error rates [12].

The use of these systems deletes hand-written transcriptions, which improves the precision, completeness, and legibility of orders and increases the possibility of accessing them from any health center [13]. A decision support system and CPOE reduces errors and increases cost-effectiveness of medical orders $[\underline{13}, \underline{14}]$.

Diabetes, one of the most common non-communicable diseases (NCDs), is the most common disease resulting from metabolic disorders and is the fourth cause of morbidity and mortality in western countries [15]. The adverse outcomes of diabetes are ocular complications, peripheral vascular disease, cardiovascular disease, neuropathy, nephropathy, and diabetic foot syndrome. Diabetes and the resulting complications are common causes of early death in most countries $[\underline{16}, \underline{17}]$.

Health expenditure on diabetes accounted for $11.6 \%$ of the total health expenditure worldwide in 2015. With such a high burden, diabetes is an obstacle to sustainable economic development. On the other hand, this disease imposes a heavy financial burden on patients and healthcare systems, for example, the average inpatient cost per patient is about

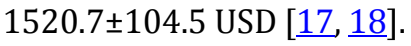

In Iran, the number of diabetic patients was estimated at 2.43 million cases in 2009 with a financial burden of about $\$ 3.78$ billion. Iran ranks the third in the total number of adult population with diabetes across the Middle East and North Africa Region (MENA). The national cost of productivity reduction following diabetes and the resulting temporary and permanent disability and productivity reduction due to early death is estimated at 1.37 billion USD in Iran $[\underline{11}, \underline{16}, \underline{19}]$.

In the past, different software engineering techniques and tools were used for analyzing healthcare systems. Currently, the concept of object oriented analysis and design using the UML is a new tool for modeling healthcare systems. UML is an object oriented modeling language that uses several visual diagrams for describing structural and behavioral features of a system $[20,21]$. The WHO has called for global action against diabetes, which should be considered an alarm for developing countries. Since establishing a diabetes information system is a priority for the Iranian Ministry of Health and Medical Education, we decided to design a computerized order system for type 2 diabetic patients by developing a conceptual model.

\section{MATERIALS AND METHODS}

This applied, descriptive, cross-sectional study was conducted in 2017.
Phase 1: Examining different modalities of designing a CPOE system following (electronic) library studies. A search was conducted in Yahoo, Google, and Google Scholar databases as well as PubMed, ISI Web of Science, and Scopus data banks.

Phase 2: In this phase, the diabetes medical records of different centers like American Diabetes Association, University of Texas, University of Illinois, Diabetes Center of Oregon University, Rocky Mountain Diabetes and Osteoporosis Center in Washington, Australia were reviewed and then a questionnaire was developed accordingly.

Phase 3: Providing a questionnaire to create a minimum data set for patient medical records based on scientific documents and patient medical records. The questionnaire contained 47 close-ended and 2 openended questions for new comments, including demographic and clinical (disease and facilitating conditions) information as well as lab tests. Content validity methods were used to validate the questionnaire, and its reliability was evaluated by pretest/posttest and a correlation of $86 \%$ was achieved.

Phase 4: Sending the checklist to an endocrinologist. The research population comprised 142 subjects. The sample size was calculated using the Cochran formula $(n=57)$.

The questionnaires were either sent by e-mail or delivered in person, and reminders were sent after a few days. After several follow-up sessions, only 43 endocrinologists completed the questionnaires. The scores were analyzed using the Microsoft Excel and Likert method. The survey was conducted using the Delphi technique in a single step because more than $75 \%$ of questions were answered with "Quite Agree" and "Agree".

The information elements of the questionnaire were approved with a minimum score of $80 \%$. Moreover, the type 2 diabetes protocol devised by the Health Deputy of the Iranian Ministry of Health and Medical Education in 2011 was used to create a minimum data set for type 2 diabetic patients. This protocol includes introduction of diabetes mellitus disease, situation of Iran, and programs for control and prevention of diabetes in urban domain [16]. This protocol was used for designing of drug databank

Finally, non-commercial UML 2.0, which is an open source software program, was used to draw UML diagrams. Then, a CPOE system was designed for patients with type 2 diabetes mellitus. The present software was prepared using the C\# programming language and SQL R2 was used for databases. 


\section{Software requirements}

The first step was to determine a minimum data set of patient records used as the patient profile in the system, including demographic and clinical information. The minimum data set in this study referred to information on level 1 healthcare centers. The information elements are demonstrated in Table 1 and Table 2 .

Table 1: Minimum data set of patient records (demographic element)

\begin{tabular}{|l|c|c|c|c|c|c|c|c|c|c|}
\hline \multirow{2}{*}{ Demographic Information } & \multicolumn{2}{|c|}{ Quite Agree } & \multicolumn{2}{|c|}{ Agree } & \multicolumn{2}{c|}{ Neutral } & \multicolumn{2}{c|}{ Disagree } & \multicolumn{2}{c|}{ Quite disagree } \\
\cline { 2 - 13 } & $\mathrm{F}$ & $\%$ & $\mathrm{~F}$ & $\%$ & $\mathrm{~F}$ & $\%$ & $\mathrm{~F}$ & $\%$ & $\mathrm{~F}$ & $\%$ \\
\hline Full name & 41 & 95 & 2 & 5 & - & - & - & - & - & - \\
\hline National ID No. & 30 & 70 & 5 & 12 & 5 & 12 & - & - & 3 & 7 \\
\hline medical record No. & 39 & 91 & 3 & 7 & - & - & - & - & 1 & 2 \\
\hline Age & 43 & 100 & - & - & - & - & - & - & - & - \\
\hline Sex & 42 & 98 & 1 & 2 & - & - & - & - & - & - \\
\hline Address & 31 & 72 & 8 & 19 & 2 & 5 & 1 & 2 & 1 & 2 \\
\hline Tel/Mobile & 39 & 91 & 4 & 9 & - & - & - & - & - & - \\
\hline Education & 32 & 74 & 8 & 19 & 3 & 7 & - & - & - & - \\
\hline Height & 40 & 93 & 2 & 5 & 1 & 2 & - & - & - & - \\
\hline Weight & 41 & 95 & 1 & 2 & 1 & 2 & - & - & - & - \\
\hline Waist circumference & 36 & 84 & 5 & 12 & 1 & 2 & 1 & 2 & - & - \\
\hline
\end{tabular}

Table 2: Minimum data set of patient records (historical element)

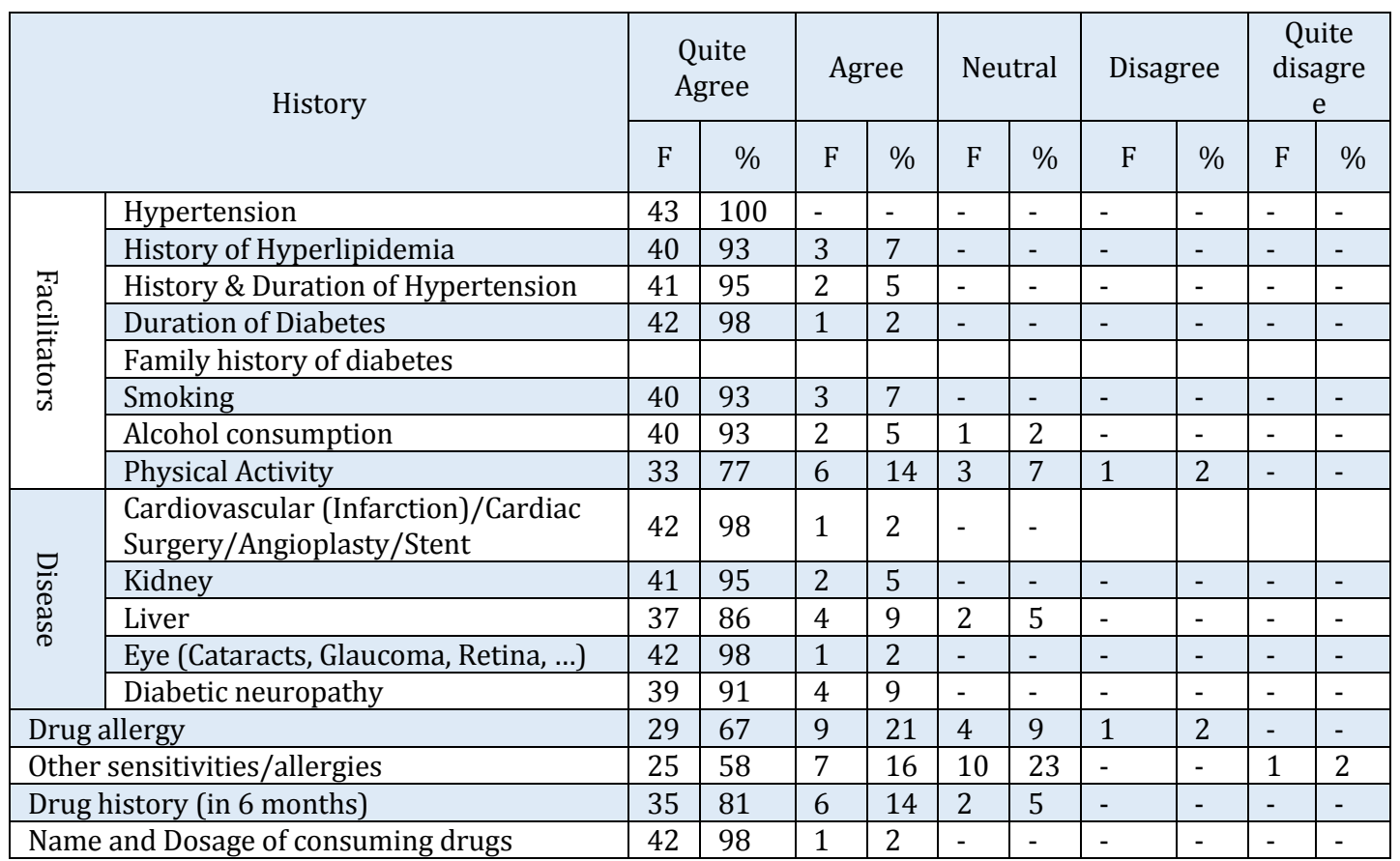

Then, laboratory tests that were required for these patients were determined through a review of the literature review and a survey of specialists.

In the second step, with use of the type 2 diabetes protocol, a drug databank was designed to instruct the physician through connecting to a decision support system in order to enhance the efficiency of prescribed drugs and quality of treatment. A list of country specific drugs and their side effects, contraindications, and dosages was used to create the databank. The list of country specific drugs for type 2 diabetic patient included Glyburide (glibenclamide) $5 \mathrm{mg}$, Glipizide 5 $\mathrm{mg}$, Glimepiride $1 \mathrm{mg}, 2 \mathrm{mg}, 3 \mathrm{mg}$, and $4 \mathrm{mg}$, Gliclazide 
$80 \mathrm{mg}$, Repaglinide (novonorm) $0.5 \mathrm{mg}, 1 \mathrm{mg}$, and 2 $\mathrm{mg}$, Nateglinide $60 \mathrm{mg}$ and $120 \mathrm{mg}$, Metformin $500 \mathrm{mg}$, Rosiglitazone $4 \mathrm{mg}$ and $8 \mathrm{mg}$, Pioglitazone $15 \mathrm{mg}, 30 \mathrm{mg}$, and 45mg, Sitagliptin Phosphate $100 \mathrm{mg}$, Exenatide 5 $\mathrm{mg}$, Miglitol, and Acarbose $50 \mathrm{mg}$ and $100 \mathrm{~m}$.

The final step was designing the software and user interface for prescribing and viewing the orders. In this section, based on Phase1,2 functional requirements of the CPOE ibility of entering simple and complicated orders, choosing pre-defined orders, entering any order, linking the pharmacy information system and other information systems, accessing recorded orders, possibility of editing orders, automatic exit, controlling drug interactions, drug allergies, repeated orders, dosage control, controlling conflicts based on patient information, controlling order parts, possibility of managing decision support rules, possibility reminders for time of entering orders, physician's interaction with the decision support system, facilitating the entry of secondary orders, automatic display of laboratory test results relevant to the order, accessibility of preselected options, providing reports from decision support activities, providing electronic guidelines, and possibility of entering date, time and digital signature by physician.

\section{UML diagrams}

Based on the minimum information elements of type 2 diabetic patients, their drug list, and functional requirements of the CPOE system for type 2 diabetes mellitus, the researcher drew UML diagrams (use case, activity, class, sequence). Then, the software was designed based on the diagrams.

Accurate analysis and exploration of model elements according to operational requirements and standards followed by developing a conceptual model are one of the essential steps in the development and analysis of information systems. Scenario preparation is the first step in system analysis. The general scheme of the CPOE system for patients with type 2 diabetes mellitus is explained in the scenarios. Each scenario consists of the use case name, description of system activities, system users, pre and post situation of use cases, and mainstream of use case. In this study, these scenarios were provided: system entry, order entry, sending orders, editing, deleting and adding orders, digital signature, automatic display of laboratory test results, display of orders sets, display of electronic guidelines, reporting decision support activities, managing decision support rules, and system exit. For example, the order entry scenario is demonstrated in Table 3.

A use case diagram represents the system functions and that is used for identifying the system's needs. In other words, it defines the operational requirements of the system. All use case diagrams included entering the system, order entry, controlling orders like drug interactions, drug allergies and conflicts based on patient information, drug dosage, controlling secondary orders, executing orders, sending orders, editing orders, automatic lab results display, display of recorded orders and orders sets, display of electronic guidelines, reporting decision support activities, managing decision support rules, digital signature, and exiting the system. For example the use-case diagrams of order entry are demonstrated in Fig 1.

Table 3: Example scenario (order entry scenario)

\begin{tabular}{|l|l|}
\hline $\begin{array}{l}\text { Use Case ID and } \\
\text { Name }\end{array}$ & UC2 :Order Entry \\
\hline Description & $\begin{array}{l}\text { Physician can entry any clinical order } \\
\text { through this use case. }\end{array}$ \\
\hline Actors & Physician \\
\hline Pre-condition & $\begin{array}{l}\text { Physician should Log in and enter the } \\
\text { system. }\end{array}$ \\
\hline Flow of Events & $\begin{array}{l}\text { Physician chooses "order entry". } \\
\text { System shows the patient list. } \\
\text { Physician chooses the special patient. } \\
\text { If this is the first order, system displays } \\
\text { the order entry page for recording } \\
\text { clinical patient information like } \\
\text { diagnosis, disease and drug history, ... } \\
\text { System shows order entry page and } \\
\text { patient information (like identity and } \\
\text { clinical information). } \\
\text { System reminds physician some } \\
\text { information like drug allergies, disease } \\
\text { history, ... } \\
\text { Physician enters title for the order. } \\
\text { If possible, some order details are filled } \\
\text { by default. } \\
\text { Physician enters the rest of order details. } \\
\text { Physician completes his entry. } \\
\text { Physician records his digital signature in } \\
\text { the system. } \\
\text { Physician clicks "Submit". } \\
\text { All order sections (including the details) } \\
\text { are controlled by the system. } \\
\text { The system adds the last order to the list } \\
\text { of orders. }\end{array}$ \\
\hline Alternate Flows & $\begin{array}{l}\text { In step 8, physician can change any of the } \\
\text { default amounts. }\end{array}$ \\
\hline Post-Conditions & $\begin{array}{l}\text { The recorded order is displayed in the } \\
\text { list of orders. }\end{array}$ \\
\hline &
\end{tabular}

Activity diagrams for entering the system, order entry, digital signature, sending orders, controlling orders, editing, deleting and adding orders, automatic display of lab results, display of recorded orders, display of orders sets, managing decision support rules, reporting clinical decision support activities, and exiting the system were drawn in this study. The order entry activity diagram is demonstrated in Fig 2 as an example.

Sequence diagrams are interaction diagrams that represent how and in what order objects operate with one another. A sequence diagram shows object interactions arranged in a time sequence. A system sequence diagram was also designed based on the same procedure. The order entry sequence diagram is 
demonstrated in Fig 3 as an example.

A class diagram is a very important diagram in UML diagrams. This diagram describes the constant structure of the system. A complete class diagram for the system is shown in Fig 4.

\section{Software development}

The final software consisted of a page for login, a page for patient list, a page for individual information, a page for history of disease and care, a page for alerting the physician about drug-drug interactions, a page for alerting the physician about disease- drug interactions, and a page for alerting the physician about drug- test results interactions.

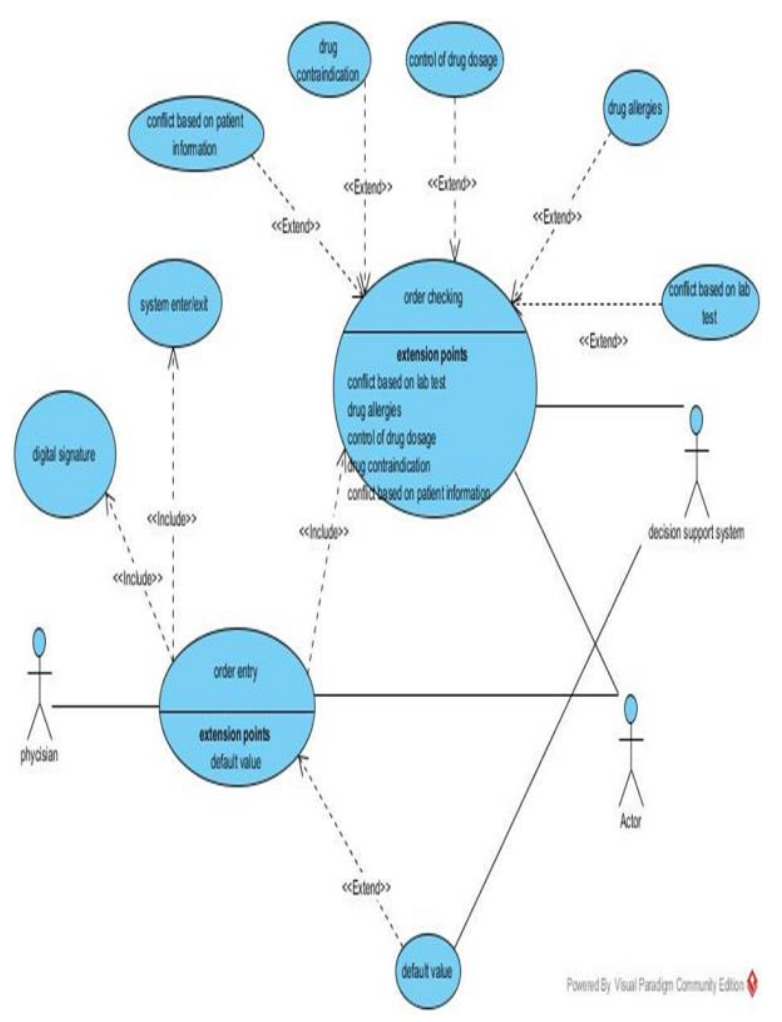

Fig 1: Use-case diagram of order entry

\section{DISCUSSION}

Software requirements for designing CPOE have different parts. The most important part of designing a system is the structure and the content of set of orders that contribute to a structured body of clinical knowledge. This part is significant compared to other parts of the software and is a strong tool to instruct physicians to execute their best performance resulting in reduced medical errors and improved care standards for patients $[\underline{14}, \underline{22}]$.

In designing a CPOE system, classifying the drugs according to their names, determined dosage, consumption dosage, consumption frequency, contraindications, drug interactions, and side effects is necessary for optimum implementation of the system. In this system, classification of all drug categories was done and the mentioned elements were included and used as a drug data bank. According to a review of the literature, nine drug categories including 22 drugs together with insulin are used in type 2 diabetes.

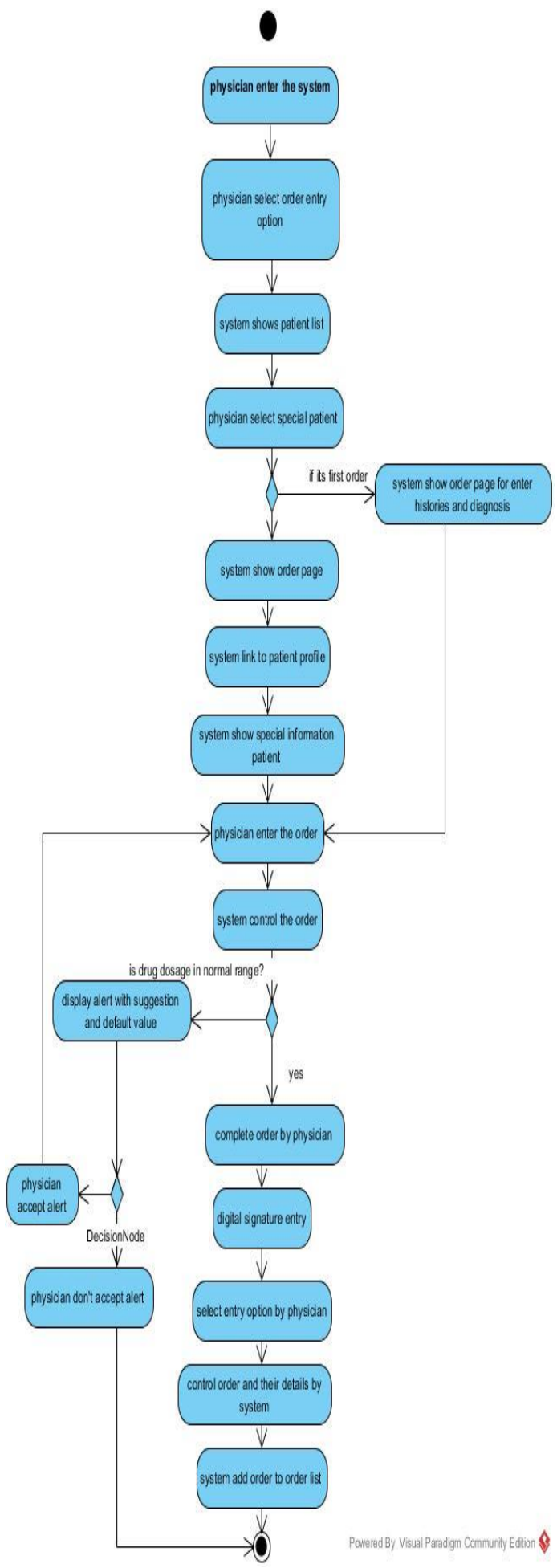

Fig 2: Order entry activity 


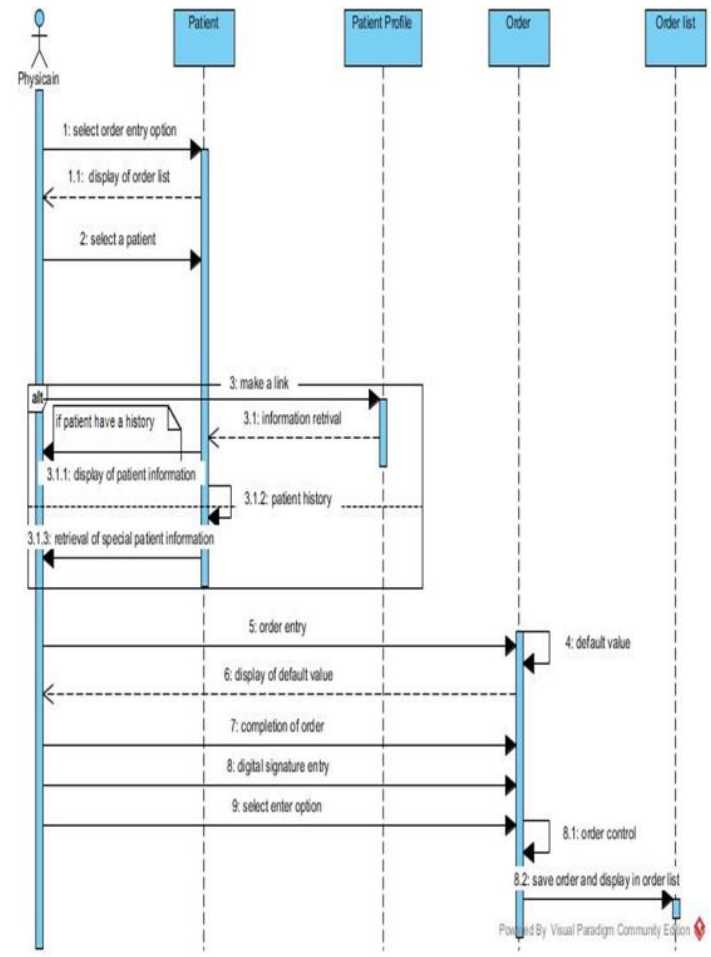

Fig 3: Order entry sequence

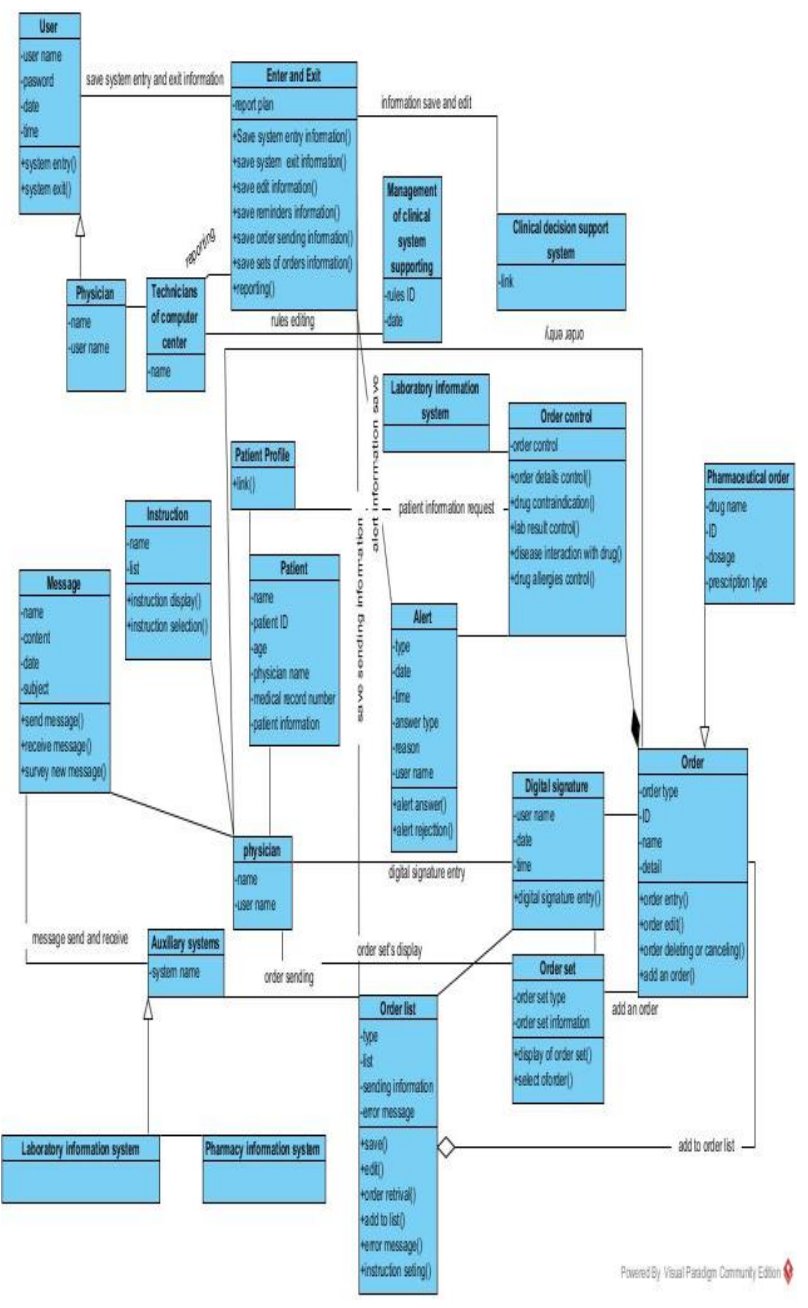

Fig 4: Complete system class
A minimum data set as a conceptual framework includes information on care effectiveness and is considered as a basis for effectiveness indicators. In particular, diabetes, which is a disease whose progress can be simply measured due to its quantitative and

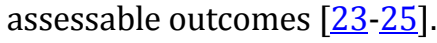

In other studies, the information elements of medical records include demographic data, general practitioner data, drug data, data of drug allergies and side effects, diabetes data including data of diabetes type 1, gestational diabetes, medical and surgical history, data of clinical tests and appointments, data of preventive and nutritional services as well as specialized eye and foot care data [26]. The findings of this study showed that the minimum data required for patients included demographics, history (disease and facilitating conditions), lab tests, and recommendations.

In other studies, the main actors are attending professors and residents, and information systems of radiology, pharmacy, nursing, laboratory and other clinical and administrative systems as well as patient profile are regarded as other actors. Moreover, operations included in these diagrams encompass the relationships between the care team, care project reporting, order entry, order set display, order history, order edit and submission, which are considered for a general educational/clinical center $[\underline{27}, \underline{28}]$. In the present study, physician was the main actor of the system and patient profile, drug store systems, laboratory system, and decision support system were the additional actors, and operations included in the diagrams were order entry, display of order sets and previous orders, log in/log out, order edit/delete/pause/add, digital signature, and order submission.

Since correct extraction of classes has a key role in the appropriate design of a conceptual model and successful analysis of an object-oriented design [28, 29], we analyzed available information and cases and drew a complete conceptual class including all properties and features, data types and classes, and their relationship with the CPOE system of type 2 diabetes mellitus [ $\underline{30}-\underline{32}]$.

\section{CONCLUSION}

A successful information system requires an appropriate design. Designing an information system is vital for every organization because accessing accurate, precise, timely and comprehensive data in the shortest possible time is a key to success [24, 30]. However, studies have shown that even advanced electronic systems cannot guarantee many information requirements; therefore, prior to designing electronic systems in clinical environments supporting information search for patient care, it is essential to understand the information requirements of the users [으. 
Hence, a CPOE system should be designed with minimum errors in recording documentations while the physician enters orders; on the other hand, offering timely information on allergens, drug interactions, side effects, and contraindications would reduce medical errors, especially drug errors, improve physician efficiency and patient satisfaction, and eventually enhance the service quality $[\underline{31}, \underline{32}]$.

\section{AUTHOR'S CONTRIBUTION}

All the authors approved the final version of the manuscript.

\section{CONFLICTS OF INTEREST}

The authors declare no conflicts of interest regarding the publication of this study.

\section{FINANCIAL DISCLOSURE}

No financial interests related to the material of this manuscript have been declared.

\section{REFERENCES}

1. Cox AR, Ferner RE. Prescribing errors in diabetes. The British Journal of Diabetes \& Vascular Disease. 2009; 9(2): 84-8.

2. Pennsylvania Patient Safety Authority. Medication errors with the dosing of insulin: Problems across the continuum. Pennsylvania Patient Safety Advisory. 2010; 7(1): 17-9.

3. Moghaddasi H, Sheikhtaheri A, Hashemi N. Reducing medication errors: Role of computerized physician order entry system. Journal of Health Administration. 2007; 10(27): 57-67.

4. Ariaei M, Nejad AS, Kouti J, Mehdipour Z, Bahaadinbeigy $\mathrm{K}$. Role of clinical decision supporting systems in prevention of medical errors from the perspective of health care staff in university hospitals of Kerman university of medical sciences. Health Information Management. 2012; 9(5): 712-23.

5. Mansouri A, Ahmadvand A, Hadjibabaie M, Javadi M, Khoee SH, Dastan F, et al. A review of medication errors in iran: sources, underreporting reasons and preventive measures. Iran J Pharm Res. 2014; 13(1): 3-17. PMID: 24734052 [PubMed]

6. Steele AM, DeBrow M. Efficiency gains with computerized provider order entry. In: Henriksen $\mathrm{K}$, Battles JB, Keyes MA (eds.). Advances in Patient Safety: New Directions and Alternative Approaches (Vol. 4: Technology and Medication Safety). Rockville (MD): Agency for Healthcare Research and Quality (US); 2008.

7. Galanter W, Falck S, Burns M, Laragh M, Lambert BL. Indication-based prescribing prevents wrong-patient medication errors in computerized provider order entry (CPOE). J Am Med Inform Assoc. 2013; 20(3): 477-81. PMID: 23396543 DOI: 10.1136/amiajnl-2012-001555 [PubMed]

8. Agrawal A. Medication errors: prevention using information technology systems. Br J Clin Pharmacol. 2009; 67(6): 681-6. PMID: 19594538 DOI: 10.1111/j.1365-2125.2009.03427.x [PubMed]

9. Khajouei R, Jaspers M. The impact of CPOE medication systems' design aspects on usability, workflow and medication orders. Methods Inf Med. 2010; 49(1): 3-19. PMID: 19582333 DOI: 10.3414/ME0630 [PubMed]

10. Nuckols TK, Smith-Spangler C, Morton SC, Asch SM, Patel VM, Anderson LJ, et al. The effectiveness of computerized order entry at reducing preventable adverse drug events and medication errors in hospital settings: A systematic review and meta-analysis. Syst Rev. 2014; 3: 56-68. PMID: 24894078 DOI: 10.1186/2046-4053-3-56 [PubMed]

11. Sharifi N, Alipour A, Ranjbar K. The effect of modern educational strategies in reducing intravenous drug administration error: A non-randomized clinical trial. Iranian Journal of Medical Education. 2012; 11(6): 5909.

12. Kaushal R, Shojania KG, Bates DW. Effects of computerized physician order entry and clinical decision support systems on medication safety: A systematic review. Arch Intern Med. 2003; 163(12): 1409-16. PMID: $12824090 \quad$ DOI: 10.1001/archinte.163.12.1409 [PubMed]

13. Levick DL, Stern G, Meyerhoefer CD, Levick A, Pucklavage D. Reducing unnecessary testing in a CPOE system through implementation of a targeted CDS intervention. BMC Med Inform Decis Mak. 2013; 13: 4350. PMID: 23566021 DOI: 10.1186/1472-6947-13-43 [PubMed]

14. Doolan DF, Bates DW. Computerized physician order entry systems in hospitals: Mandates and incentives. Health Aff (Millwood). 2002; 21(4): 180-8. PMID: 12117128 DOI: $10.1377 /$ hlthaff.21.4.180 [PubMed]

15. Esteghamati A, Larijani B, Aghajani MH, Ghaemi F, Kermanchi J, Shahrami A, et al. Diabetes in Iran: Prospective analysis from first nationwide diabetes report of national program for prevention and control of diabetes (NPPCD-2016). Sci Rep. 2017; 7(1): 1346171. PMID: 29044139 DOI: 10.1038/s41598-017-13379$\mathrm{z}$ [PubMed]

16. Valizadeh R, Vali L, Bahaadinbeigy K, Amiresmaili M. The challenges of Iran's type 2 diabetes prevention and control program. Int J Prev Med. 2019; 10(1): 175.

17. World Health Organization. Global report on diabetes. 2016.

18. Farshchi A, Esteghamati A, Sari AA, Kebriaeezadeh A, Abdollahi M, Dorkoosh FA, et al. The cost of diabetes chronic complications among Iranian people with type 2 diabetes mellitus. J Diabetes Metab Disord. 2014; 13(1): 42-56. PMID: 24593991 DOI: 10.1186/2251-6581-1342 [PubMed]

19. Riazi H, Larijani B, Langarizadeh M, Shahmoradi L. Managing diabetes mellitus using information technology: A systematic review. J Diabetes Metab 
Disord. 2015; 14: 49-58. PMID: 26075190 DOI: 10.1186/s40200-015-0174-x [PubMed]

20. Dennis A, Wixom BH, Tegarden D. Systems analysis and design: An object-oriented approach with UML. John Wiley \& Sons; 2015.

21. Evans A, France R, Lano K, Rumpe B. Developing the UML as a formal modelling notation. In: Pierre-Alain Muller, Jean Bezivin (eds.). UML'98 eyond the notation. International Workshop Mulhouse France. Universite de Haute-Alsace. 1998.

22. deLusignan S. Benefits and disbenefits of the implementation and adoption of computerised medical records (CMR); plus a more accessible approach to modelling. BMJ Health Care Informatics. 2016; 23(1): iii.

23. Hosseini A, Moghaddasi H, Jahanbakhsh M. Designing minimum data sets of diabetes mellitus: Basis of effectiveness indicators of diabetes management. Health Information Management. 2010; 7(3): 330-40.

24. Ahmadi M, Ghazisaeidi M, Bashiri A. Radiology reporting system data exchange with the electronic health record system: A case study in Iran. Glob J Health Sci. 2015; $\begin{array}{lccc}\text { 7(5): 208-14. PMID: } 26156904 & \text { DOI: } \\ \text { 10.5539/gjhs.v7n5p208 [PubMed] } & \end{array}$

25. Riazi H, Langarizadeh M, Larijani B, Shahmoradi L. Conceptual framework for developing a diabetes information network. Acta Inform Med. 2016; 24(3): $\begin{array}{lccc}\text { 186-92. PMID: } & \text { 27482133 } & \text { DOI: } \\ \text { 10.5455/aim.2016.24.186-192 } & \text { [PubMed] }\end{array}$

26. Jung E-Y, Kim J, Chung K-Y ,Park DK. Mobile healthcare application with EMR interoperability for diabetes patients. Cluster Computing. 2014; 17(3): 871-80.

27. Amanzadeh M. Conceptual model of CPOE. [Thesis] Shahid Beheshti University: Tehran; 2010.

28. Samadbeik M. Conceptual model for a national system of electronic prescribing. [Thesis] Tehran University of Medical Sciences: Tehran; 2013.

29. Ahlan AR, Ahmad BI. An overview of patient acceptance of health information technology in developing countries: A review and conceptual model. International Journal of Information Systems and Project Management. 2015; 3(1): 29-48.

30. Shajarat M. Assessment of individual EHR information elements of diabetes base on mobile. [Thesis]. Tehran University of Medical Science: Tehran; 2012.

31. Sadeghi F. The design of a reconstructive hand surgery text database based on a speech recognition system. [Thesis]. Tehran University of Medical Science: Tehran; 2013.

32. Coustasse A, Shaffer J, Conley D, Coliflower J, Deslich S, Sikula A. Computer Physician Order Entry (CPOE): Benefits and concerns-a status report. Journal of Information Technology Research. 2013; 6(3): 16-31. 